\title{
Piperaquine concentration and malaria treatment outcomes in Ugandan children treated for severe malaria with intravenous Artesunate or quinine plus Dihydroartemisinin-Piperaquine
}

Pauline Byakika-Kibwika ${ }^{1,2^{*}}$, Ronald Ssenyonga ${ }^{3}$, Mohammed Lamorde ${ }^{2}$, Daniel Blessborn ${ }^{4,5}$ and Joel Tarning ${ }^{4,5}$

\begin{abstract}
Background: Treatment for severe malaria must be prompt with effective parenteral antimalarial drugs for at least $24 \mathrm{~h}$ to achieve fast parasite clearance, and when the patient can tolerate oral therapy, treatment should be completed with effective artemisinin based combination therapy (ACT) for complete parasite clearance and to prevent recrudescence. We evaluated piperaquine concentration and malaria treatment outcomes among Ugandan children treated for severe malaria with intravenous artesunate (AS) or quinine (QN) plus dihydroartemisininpiperaquine (DP), in Tororo District Hospital in Eastern Uganda.

Methods: Capillary blood piperaquine concentration data were obtained from a randomized clinical trial whose objective was to evaluate parasite clearance, 42-day parasitological treatment outcomes and safety, following treatment of severe malaria with intravenous AS or QN, plus artemether-lumefantrine or DP among children in Tororo District Hospital, in Eastern Uganda.

Results: Piperaquine concentration data from 150 participants who received DP were analyzed. Participants with unadjusted treatment failure had lower median day 7 capillary piperaquine concentration than those with treatment success (34.7 (IQR) (17.9-49.1) vs 66.7 (IQR) (41.8-81.9), $p<0.001)$, and lower than the recommended day 7 cut off level of $57 \mathrm{ng} / \mathrm{mL}$. There was no difference in median capillary piperaquine concentrations among participants with re-infection and recrudescence (35.3 (IQR) (17.9-55.2) vs 34.8 (IQR) (18.1-45.1), $p=0.847)$. The risk of treatment failure was two times higher among children with low $(<57 \mathrm{ng} / \mathrm{mL})$ day 7 capillary piperaquine concentration (relative risk: $2.1 \mathrm{Cl} 1.4-3.1), p<0.001$ ) compared to children with high day 7 capillary piperaquine concentrations (> $57 \mathrm{ng} / \mathrm{mL}$ ).

Conclusion: Considering the low day 7 concentrations of piperaquine reported in the patients studied here, we suggest to adopt the recently recommended higher dose of DP in young children or a prolonged 5-day dosing in children living in malaria endemic areas who have suffered an initial episode of severe malaria in order to achieve adequate drug exposures for effective post-treatment prophylactic effects.
\end{abstract}

Trial registration: The study was registered with the Pan African Clinical Trial Registry (PACTR201110000321348). Registered 7th October 2011.

Keywords: Piperaquine, Pharmacokinetics, Malaria, Children

\footnotetext{
* Correspondence: pbyakika@gmail.com

${ }^{1}$ Department of Medicine, Makerere University College of Health Sciences,

Kampala, Uganda

${ }^{2}$ Infectious Diseases Institute, Kampala, Uganda

Full list of author information is available at the end of the article
}

(c) The Author(s). 2019 Open Access This article is distributed under the terms of the Creative Commons Attribution 4.0 International License (http://creativecommons.org/licenses/by/4.0/), which permits unrestricted use, distribution, and reproduction in any medium, provided you give appropriate credit to the original author(s) and the source, provide a link to the Creative Commons license, and indicate if changes were made. The Creative Commons Public Domain Dedication waiver (http://creativecommons.org/publicdomain/zero/1.0/) applies to the data made available in this article, unless otherwise stated. 


\section{Background}

Severe malaria is a life threatening emergency, responsible for 435,000 deaths annually, worldwide, with the greatest burden in sub-Saharan Africa [1]. Treatment for severe malaria must be prompt with effective parenteral antimalarial drugs for at least $24 \mathrm{~h}$ to achieve fast parasite clearance, and when the patient can tolerate oral therapy, treatment should be completed with effective artemisinin based combination therapy (ACT) for complete parasite clearance and to prevent recrudescence. Uganda adopted the policy to use intravenous artesunate (AS) as first line treatment for severe malaria in 2013, with intravenous quinine (QN) or intramuscular artemether as alternatives. The oral ACTs; artemetherlumefantrine $(\mathrm{AL})$ and dihydroartemisinin-piperaquine (DP) are recommended for complete parasite clearance [2-4]. Although previous studies demonstrated excellent effectiveness of both AL and DP for treatment of uncomplicated malaria, DP provides the additional advantage of once daily dosing and longer post treatment prophylactic effect of up to 35 days compared to AL, which is dosed twice daily and with a shorter post treatment prophylactic period of 28 days $[3,5,6]$. We evaluated capillary piperaquine concentration and malaria treatment outcomes among Ugandan children treated for severe malaria with intravenous AS or QN plus DP, in Tororo District Hospital in Eastern Uganda.

\section{Methods}

\section{Study design, site and population}

Study methodology has been previously described and published as a randomized single blind clinical trial conducted in Tororo District Hospital in Eastern Uganda [7], an area with perennial malaria transmission and an annual entomological inoculation rate estimated to be 310 infective bites per person per year [8]. The main study enrolled consecutive patients aged 6 months and above, with signed informed consent provided by the parent or guardian and severe malaria defined as presence or history of fever plus a positive blood film for P.falciparum malaria, with at least one of the laboratory or clinical features of severe malaria. Patients were excluded if they had obvious concomitant febrile illness, history of allergy to any of the study drugs, if they could not comply with study procedures and visits, or if they had received an antimalarial drug within $24 \mathrm{~h}$ before presenting to hospital.

\section{Treatment}

Intravenous AS (Guilin Pharmaceutical Factory, Guangxi, China) was administered as a slow bolus into an indwelling cannula as $2.4 \mathrm{mg} / \mathrm{kg}$ at start of treatment, repeated at 12 and $24 \mathrm{~h}$ and every $24 \mathrm{~h}$ till the switch to oral therapy. Intravenous QNN dihydrochloride (Rotex,
Trittau, Germany) was administered over $4 \mathrm{~h}$ as $10 \mathrm{mg} /$ $\mathrm{kg}$ body weight in $5 \%$ dextrose $(10 \mathrm{ml} / \mathrm{kg})$ and repeated 8 hourly till the switch to oral therapy.

Parenteral antimalarial therapy was administered for at least $24 \mathrm{~h}$, followed by a full course of the oral ACT when participants could tolerate oral therapy. Oral AL (Coartem, Novartis, $20 \mathrm{mg}$ artemether/120 mg lumefantrine tablets) was administered according to body weight as; one (5-14 kg), two (15-24 kg), three $(25-34 \mathrm{~kg})$ and four (> $35 \mathrm{~kg}$ ) tablets 12 hourly, with a cup of milk or food, for 3 days. Oral DP (Eurartesim, Sigma-Tau, dihydroartemisinin (DHA) $40 \mathrm{mg}+$ piperaquine (PQP) 320 mg tablets) was administered targeting a total dose of 6.4 and $51.2 \mathrm{mg} / \mathrm{kg}$ of dihydroartemisinin and piperaquine, respectively, given in three equally divided doses to the nearest quarter tablet. We used a pill cutter to ensure that the tablet fractions were as close to the nearest quarter tablet as possible.

All participants received oral paracetamol in a dose of $15 \mathrm{mg} / \mathrm{kg}$ at 8 hourly intervals. Adjunctive and supportive treatment for complications of malaria such as convulsions and hypoglycemia was given in accordance with the Uganda Ministry of Health guidelines. The study nurse provided information to caretakers about adherence to drugs, follow-up visits and potential drug side effects. Caretakers were instructed to observe the participants for $30 \mathrm{~min}$ after drug administration and if vomiting occurred they were to administer another dose, for up to two extra doses, following which they were to bring back the participant to the study clinic for evaluation and treatment.

\section{Follow-up}

We performed serial blood smears at $0,1,2,4,6,8,10$, $12,16,20,24 \mathrm{~h}$ post start of intravenous therapy and every $6 \mathrm{~h}$ until $6 \mathrm{~h}$ post parasite clearance. Participants were initiated on oral ACT and discharged from hospital when they could tolerate oral ACT and the blood smear was negative for malaria parasites, and were followed up for 42 days to ascertain parasitological outcomes and monitor adverse events on days 1, 2, 3, 7, 14, 21, 28, 35, 42 , and any unscheduled day if the participant felt unwell. On each of these days we took medical history and performed physical examination, a finger prick was done to collect blood on slides for malaria diagnosis and on filter paper for genotyping and drug concentration measurements. Participants with positive malaria films were reassessed for severity and treated accordingly, those with severe malaria were re-admitted and treated with intravenous AS plus AL, and those with uncomplicated malaria were evaluated for treatment failure and treated according to national guidelines. Participants were discontinued from study follow up if they could not take study medication, missed a scheduled follow-up visit and 
could not be located at home, or if they received non study drugs.

\section{Laboratory procedures}

Thin smears were performed to determine the type of malaria parasite species and thick smears for parasite density. Thick blood smears were stained using 3\% Giemsa for $30 \mathrm{~min}$ and read by two independent experienced laboratory technologists, blinded to participants' treatment assignment. Any discrepant results were reviewed by a tie breaker.

Parasite density was calculated by counting the number of asexual parasites (ring stages) per 200 white blood cells (WBCs) or per 500 if the count was less than 10 parasites per $200 \mathrm{WBCs}$, assuming a WBC count of $8000 / \mathrm{uL}$ of blood. A smear was considered negative if no parasites were seen after review of 100 high-power fields. Complete blood count and hemoglobin estimation were performed using the Coulter counter (Beckman coulter, Life Science, United States of America).

Molecular genotyping of paired samples was conducted to distinguish re-infection from recrudescence, at the Makerere University-University of California San Francisco Molecular Biology laboratory in Mulago, Kampala. We used Whatman 3MM filter paper from Sigma. Parasite DNA was extracted from filter paper blood samples collected on the day of enrollment and the day of parasitological treatment failure using Chelex 100 Resin extraction (Bio-Rad Laboratories, Hercules, CA) as previously described [9]. The surface antigen loci MSP1, MSP2 and GLURP were amplified using previously described primers [10]; $2 \mu \mathrm{L}$ of template DNA was amplified using nested polymerase chain reaction (PCR), with second round primers specific to allelic families: $\mathrm{K} 1, \mathrm{MAD} 20$, and RO33 for msp1, msp2 and the repeat region of glurp [11]. PCR products were stained with ethidium bromide separated by electrophoresis on a 2.5\% agarose gel (UltraPure Agarose; Invitrogen, Carlsbad, CA). GelCompar II software (Applied Maths, SintMartens-Latem, Belgium) was used to select alleles and estimate the size of PCR products using a standardized approach [12]. Recrudescence was defined as the presence of all matched alleles on day 0 and the day of failure at every locus and reinfection defined as at least one locus showing unmatched alleles.

\section{Classification of outcomes}

The primary study outcome was parasitological treatment failure unadjusted by genotyping classified as parasitemia detected by thick blood smear. This primary outcome was selected because it best represents the treatment outcome measure used in routine clinical care. The secondary outcomes were parasitological treatment failure adjusted by genotyping classified as reinfection or recrudescence. Adverse events were defined as any medical occurrence post study drug administration. They were graded as mild, moderate, severe and life threatening and their relationship to the study drug was classified as unrelated, possibly, probably or definitely related to study drug.

\section{Piperaquine concentration measurement}

On each follow up day ie days $1,2,3,4,7,14,21,28,35$, 42 post the commencement of ACT administration, capillary blood samples were collected by finger prick and stored dry on Whatman 3MM filter paper. The fingers were disinfected and pricked with a lancet, following which the first blood drop of blood was discarded and the next three drops collected. Each drop filled a pre marked circle on filter paper. The filter papers were allowed to dry at room temperature and packed in sealed ziplock bags.

The blood samples were transported at room temperature, to the Department of Clinical Pharmacology at the Mahidol-Oxford Tropical Medicine unit, Faculty of Tropical Medicine, Mahidol University, Bangkok, Thailand. Piperaquine concentrations were measured using an LC-MS/MS based assay and validated according to U.S. FDA guidelines (unpublished data). Briefly, 3 discs of $3.2 \mathrm{~mm}$ diameter were punched out from each dried blood spot sample and $375 \mu \mathrm{L}$ stable isotope internal standard in phosphate buffer $50 \mathrm{mmol} / \mathrm{L} \mathrm{pH} 2.0$ was added followed by $150 \mu \mathrm{L}$ perchloric acid $(0.3 \mathrm{~mol})$ and $75 \mu \mathrm{L}$ acetonitrile and then mixed for $60 \mathrm{~min}$. Approximately $500 \mu \mathrm{L}$ was transferred to a 96 well plate solid phase extraction column and extracted with a MPC-SD Empore 96-wellplate standard well $1 \mathrm{ml}$ (3 M Empore, 3 M Centre, St. Paul, MN, USA). The extracted sample was then evaporated until dry and reconstituted in $250 \mu \mathrm{L}$ acetonitrile-ammonium bicarbonate $2.5 \mathrm{mmol} /$ $\mathrm{L}$ pH $10(85-15 \mathrm{v} / \mathrm{v})$. The LC-MS/MS assay settings were the same as a previously published method [13]. The lower limit of quantification was $3 \mathrm{ng} / \mathrm{ml}$ and triplicate quality control samples at low, medium and high concentration was included in each batch of samples to ensure accuracy and precision of the assay. The total coefficient of variation for all quality control samples were within the acceptance criteria of the U.S. FDA guidelines for sample analysis.

\section{Ethical considerations}

The study was approved by Makerere University School of Medicine Research and Ethics Committee (REC REF 2011-175), Uganda National Drug Authority (369/ESR/ NDA/DID-12/2011), Uganda National Council for Science and Technology (HS 1031) and registered with the Pan African Clinical Trial Registry (PACTR201110000321348). All study procedures were conducted according to Good 
Clinical Practice standards. Patients and parents or guardians of participants provided written informed consent prior to enrollment. Study related information was provided in the participants' local languages.

\section{Statistical analysis}

Data were entered and verified using MS ACCESS and analyzed using STATA version 13.1 (STATA Corporation, College Station, TX, USA). Descriptive statistics were used to compare demographic and clinical characteristics among the four study arms. Continuous variables were compared using Wilcoxon test for nonnormally distributed data. Categorical variables were compared using Chi-square test. Parasite density was normalized using logarithmic transformation. Intentionto-Treat analysis was used for comparison of treatment outcomes, which included all enrolled participants. Unadjusted treatment failure was classified as a positive blood smear on any of the follow-up days. Adjusted treatment failure was classified as either re-infection or recrudescence based on genotyping. The risk of treatment failure at 28,35 and 42 days of follow up (unadjusted and adjusted by genotyping) were estimated using the Kaplan-Meier survival method and compared using the Log Rank test. Time at risk was calculated from day one of ACT allocation to date of treatment failure among participants who failed, last day of followup for those who did not complete follow-up, or day 42 for the patients who completed 42 days of follow-up. In the analysis for adjusted parasitological outcomes, only recrudescence was considered as true parasitological treatment failure. Safety data from all participants were analyzed.

Piperaquine concentration data were compared using the Wilcoxon test. We compared day 7, 14, 35 and 42 piperaquine concentration among children with and without malaria treatment failure. Day 7 piperaquine concentration were also stratified above/below a previously reported cut off level of $57 \mathrm{ng} / \mathrm{mL}$, associated with an increased risk of therapeutic failure [9], and evaluated with respect to risk of malaria treatment failure.

\section{Results}

We enrolled and followed up 300 participants between January 2012 and March 2013, of whom, 150 received DP. Baseline characteristics were similar across the four treatment arms (Table 1). Adverse events occurred commonly, although most were of mild to moderate severity and consistent with malaria symptoms. The most common were headache, nausea and vomiting. All severe adverse events were classified as unrelated to study drugs and all were treated and resolved completely.

\section{Piperaquine concentration}

Capillary piperaquine concentration data from the 150 participants who received DP were analyzed. Of these,

Table 1 Baseline characteristics of study participants

\begin{tabular}{|c|c|c|c|c|}
\hline Characteristic & $\begin{array}{l}\text { AS+DP } \\
N=79\end{array}$ & $\begin{array}{l}\mathrm{AS}+\mathrm{AL} \\
N=71\end{array}$ & $\begin{array}{l}\mathrm{QN}+\mathrm{DP} \\
\mathrm{N}=71\end{array}$ & $\begin{array}{l}\mathrm{QN}+\mathrm{AL} \\
N=79\end{array}$ \\
\hline Female (\%) & $36(45.6)$ & $36(50.7)$ & $24(33.8)$ & $37(46.8)$ \\
\hline Age in months* & $17(11-26)$ & $16(10-26)$ & $17(13-26)$ & $18(13-26)$ \\
\hline Weight $(\mathrm{kgs})^{*}$ & $9.5(8-11.5)$ & $9(8.1-11)$ & $9.8(8.8-11)$ & $9.2(8.4-11)$ \\
\hline Temperature (degrees Centigrade)* & $38.8(37.7-39.5)$ & $39.1(37.7-39.5)$ & $39.1(37.3-39.5)$ & $38.6(37.5-39.6)$ \\
\hline Parasite density per uL, $\log 10$ copies* $^{*}$ & $4.82(4.29-5.10)$ & $4.79(4.38-5.02)$ & $4.72(4.19-5.00)$ & $4.73(4.17-5.03)$ \\
\hline \multicolumn{5}{|l|}{ Complications at admission, n (\%) } \\
\hline Hemoglobin $(\mathrm{mg} / \mathrm{dL})^{*}$ & $9.1(7.9-10.5)$ & $9.2(8.4-10.6)$ & $9.3(8.4-10.4)$ & $9.4(8.0-10.3)$ \\
\hline Total white blood cell count $\left({ }^{*} 10^{3 /} \mathrm{uL}\right)^{*}$ & $9.6(6.9-13.2)$ & $9.2(7.5-12.3)$ & $9.4(7.7-12.1)$ & $10.0(7.0-14.3)$ \\
\hline Random blood sugar (mmol/L)* & $7.3(6.3-8.3)$ & $6.8(6.4-8.3)$ & $6.8(5.7-8.3)$ & $7.4(6.25-8.25)$ \\
\hline History of repeated convulsions n (\%) & $6(7.6 \%)$ & $3(4.2 \%)$ & $8(11.3 \%)$ & $1(1.3 \%)$ \\
\hline History of inability to feed & $26(33.0 \%)$ & $24(34.0 \%)$ & $22(31.0 \%)$ & $29(36.7 \%)$ \\
\hline Prostration (extreme weakness) & $22(27.9 \%)$ & $15(21.13 \%)$ & $21(29.58 \%)$ & $21(26.58 \%)$ \\
\hline Hemoglobinuria & 0 & 0 & $2(2.8 \%)$ & 0 \\
\hline Jaundice & $2(2.5 \%)$ & 0 & $3(4.2 \%)$ & $3(3.8 \%)$ \\
\hline Severe anemia & 0 & 0 & $1(3 \%)$ & $2(2.5 \%)$ \\
\hline Respiratory distress & $2(2.5 \%)$ & $6(8.5 \%)$ & $3(4.2 \%)$ & $5(6.3 \%)$ \\
\hline Impaired consciousness & 0 & 0 & $1(1.4 \%)$ & 0 \\
\hline Abnormal bleeding & $2(2.5 \%)$ & $1(1.4 \%)$ & 0 & $1(1.3 \%)$ \\
\hline Hypoglycemia & 0 & 0 & 0 & $1(1.3 \%)$ \\
\hline
\end{tabular}


17 had treatment outcome assignment before day 7, and 133 were followed up past day 7. Median (IQR) day 7 capillary piperaquine concentration was 42.1 (23.2-67.2) $\mathrm{ng} / \mathrm{mL}$, lower than the recommended cut off level of 57 $\mathrm{ng} / \mathrm{mL}$ [9], and $90(67.7 \%)$ had capillary piperaquine concentration less than $57 \mathrm{ng} / \mathrm{mL}$.

The observed median (IQR) day 7 capillary piperaquine concentrations were significantly lower in patients with recrudescence $(34.8 \mathrm{ng} / \mathrm{mL}$ (IQR) (18.1-45.1) and re-infection (35.3 ng/mL (IQR) (17.9-55.2) compared to patients with successful malaria treatment outcome (66.7 ng/mL (IQR) (41.8-81.9), both $p<0.001$ ).

Furthermore, the risk of treatment failure (recrudescence and re-infection) was two times higher (relative risk: $2.1,95 \%$ CI: 1.4-3.1, $p<0.001$ ) among children with low $(<57 \mathrm{ng} / \mathrm{mL})$ day 7 capillary piperaquine concentrations compared to children with high day 7 capillary piperaquine concentrations $(>57 \mathrm{ng} / \mathrm{mL}$ ). Figure 1 shows the observed capillary piperaquine concentrations on each study day, stratified by study arm and Table 2 shows capillary piperaquine concentrations stratified by treatment outcome and follow up day.

\section{Discussion}

The objective of this study was to evaluate piperaquine concentration and malaria treatment outcomes among Ugandan children treated for severe malaria with intravenous AS or QN plus DP, in Tororo District Hospital in Eastern Uganda. The oral ACT, DP was effective at clearing parasites during the 42-day follow-up period, with good safety outcomes. There were low rates of recrudescence, majority of the study patients classified as treatment failures suffered re-infection with malaria parasites during the follow-up period [7]. Our findings are consistent with previous data from similar high malaria transmission settings which demonstrated high rates of re-infection with malaria parasites after initial antimalarial treatment $[2,10,11]$. The high malaria re-infection rate is particularly important in patients with severe malaria since re-infection is likely to cause further comorbidity and resulting in negative health and socialeconomic impact.

Piperaquine is the long acting partner drug in the DP combination and is responsible for clearing residual parasites to prevent recrudescence while also preventing reinfection (post treatment prophylaxis). Previous studies have reported day 7 piperaquine capillary concentration of $57 \mathrm{ng} / \mathrm{mL}$ as a therapeutic target, with lower concentrations associated with an increased risk of recrudescence in patients treated for uncomplicated malaria [14]. In 2015 Sambol et al. reported that less than 30\% of Ugandan children receiving weight based dosing of piperaquine for uncomplicated malaria achieved $57 \mathrm{ng} /$ $\mathrm{mL}$ on day 7 [15]. Our findings are in agreement with this as only $32.3 \%$ of our patients achieved more than the target of $57 \mathrm{ng} / \mathrm{mL}$.

We found that children with piperaquine concentration below this target had an approximately 2-fold higher risk of malaria treatment failure. Despite this, we demonstrated low levels of recrudescence among our study participants with no difference in the risk of recrudescence across study arms [7].

Previous studies have demonstrated high risk for readmission or death within 6 months' post discharge among children hospitalized with severe malaria in malaria endemic areas. Therefore, researchers have recommended administration of malaria chemoprevention with DP at discharge in order to protect from novel infections in the period following the severe malaria episode [16-18]. Treatment with DP offers superior post-

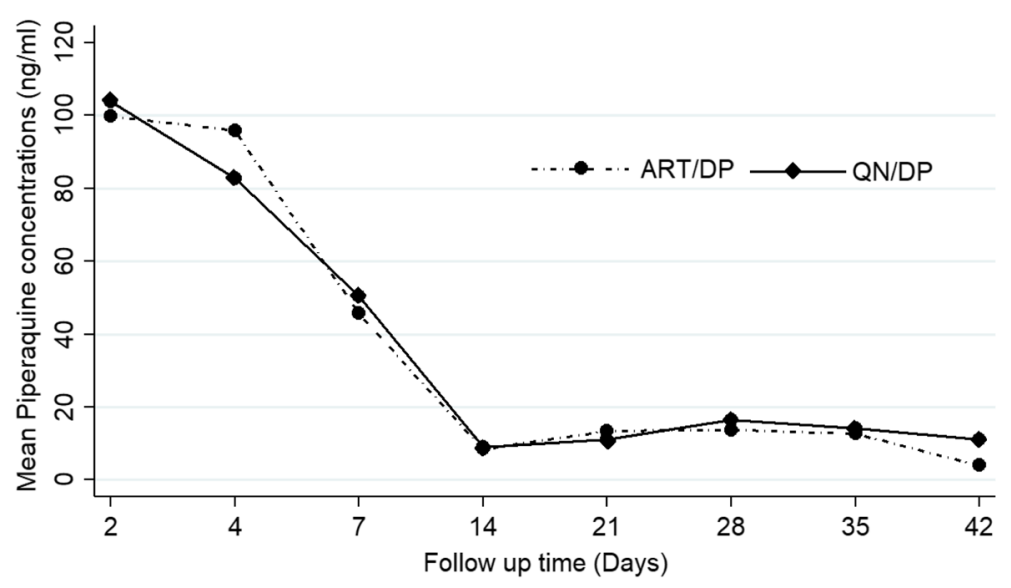

Y-axis: Piperaquine concentration $(\mathrm{ng} / \mathrm{mL})$

$\mathrm{X}$-axis: Time in days

Fig. 1 Mean (SD) capillary piperaquine concentration by study day and arm 
Table 2 Capillary piperaquine concentration, stratified by treatment outcome and day

\begin{tabular}{|c|c|c|c|c|c|c|}
\hline \multirow[t]{3}{*}{ Day } & \multicolumn{6}{|c|}{ Median (IQR) capillary piperaquine concentration (ng/mL) } \\
\hline & \multicolumn{3}{|c|}{ Unadjusted treatment outcomes } & \multicolumn{3}{|c|}{ Adjusted treatment outcomes } \\
\hline & Treatment Success $(n=52)$ & Treatment Failure $(n=98)$ & p & Re-infection $(n=63)$ & Recrudescence $(n=35)$ & $p$ \\
\hline 2 & $126(61.4-215.0)$ & $58.4(30.3-105.0)$ & 0.002 & $61.5(34.3-105)$ & $61.5(35.8-94.3)$ & 0.939 \\
\hline 4 & $110.5(77.5-155.5)$ & $66.7(32.4-103.0)$ & $<0.001$ & $72.8(38.8-106)$ & $59.4(24.3-98.9)$ & 0.300 \\
\hline 7 & $66.7(41.8-81.9)$ & $34.7(17.9-49.1)$ & $<0.001$ & $35.3(17.9-55.2)$ & $34.8(18.1-45.1)$ & 0.847 \\
\hline 28 & $15.3(11.3-23.6)$ & $10.8(7.6-14.3)$ & 0.001 & $11.4(7.3-16.7)$ & $14.3(8.2-18.1)$ & 0.549 \\
\hline 35 & $12.1(8.2-5.6)$ & $10.4(5.0-15.4)$ & 0.352 & $7.9(4.9-11.1)$ & $15.4(13.2-22.2)$ & 0.095 \\
\hline
\end{tabular}

treatment prophylaxis compared to AL, due to the longer terminal elimination half-life of piperaquine leading to therapeutic concentrations for an extended period of time compared to lumefantrine [11].

\section{Conclusion}

Considering the low day 7 concentrations of piperaquine reported in the patients studied here, we suggest to adopt the recently recommended higher dose of DP in young children [19] or a prolonged 5-day dosing in children living in malaria endemic areas who have suffered an initial episode of severe malaria in order to achieve adequate drug exposures for effective post-treatment prophylactic effects.

\section{Abbreviations}

ACT: Artemisinin based combination therapy; AL: Artemether-lumefantrine; AS: Artesunate; DHA: Dihydroartemisinin; DP: Dihydroartemisinin-piperaquine; IQR: Inter Quartile Range; PCR: Polymerase chain reaction; PQP: Piperaquine; QN: Quinine; WHO: World Health Organization

\section{Acknowledgements}

We thank the study participants and members of the clinical study team.

\section{Authors' contributions}

PBK and ML participated in study design and data collection, JT and DB performed the piperaquine assays, RS and PBK analyzed and interpreted the data, PBK drafted the first version and all authors reviewed and approved the manuscript.

\section{Funding}

This project is part of EDCTP1 program supported by the European Union (TA 2009.40200.020) and the International Society for Infectious Diseases $(077166 / Z / 05 / Z)$. The funding bodies did not have a role in the design of the study and collection, analysis, and interpretation of data and in writing the manuscript. The Mahidol-Oxford Tropical Medicine Research Unit is funded by the Wellcome Trust of Great Brittan.

\section{Availability of data and materials}

The datasets used and/or analyzed during the current study are available from the corresponding author on reasonable request.

\section{Ethics approval and consent to participate}

The study was approved by Makerere University School of Medicine Research and Ethics Committee (REC REF 2011-175), Uganda National Drug Authority (369/ESR/NDA/DID-12/2011), Uganda National Council for Science and Technology (HS 1031) and registered with the Pan African Clinical Trial Registry (PACTR201110000321348). All study procedures were conducted according to Good Clinical Practice standards. Patients and parents or guardians of participants provided written informed consent prior to enrollment. Study related information was provided in the participants' local languages. The dataset supporting the conclusions of this article is included in the submission as an additional file.

\section{Consent for publication}

"Not applicable" in this section.

\section{Competing interests}

The authors declare that they have no competing interests.

\section{Author details}

${ }^{1}$ Department of Medicine, Makerere University College of Health Sciences, Kampala, Uganda. ${ }^{2}$ Infectious Diseases Institute, Kampala, Uganda. ${ }^{3} \mathrm{Clinical}$ Trials Unit, Makerere University College of Health Sciences, Kampala, Uganda. ${ }^{4}$ Mahidol-Oxford Tropical Medicine Research Unit, Mahidol University, Bangkok, Thailand. ${ }^{5}$ Centre for Tropical Medicine, Nuffield Department of Medicine, University of Oxford, Oxford, UK.

Received: 12 July 2019 Accepted: 22 November 2019

Published online: 03 December 2019

\section{References}

1. World Health Organisation: World Malaria Report. 2018

2. Nanyunja M, Nabyonga Orem J, Kato F, Kaggwa M, Katureebe C, Saweka J. Malaria treatment policy change and implementation: the case of Uganda. Malar Res Treat. 2011;2011:683167.

3. Yeka A, Dorsey G, Kamya MR, Talisuna A, Lugemwa M, Rwakimari JB, Staedke SG, Rosenthal PJ, Wabwire-Mangen F, Bukirwa H. Artemetherlumefantrine versus dihydroartemisinin-piperaquine for treating uncomplicated malaria: a randomized trial to guide policy in Uganda. PLoS One. 2008;3:e2390

4. Arinaitwe E, Sandison TG, Wanzira H, Kakuru A, Homsy J, Kalamya J, Kamya MR, Vora N, Greenhouse B, Rosenthal PJ, et al. Artemether-lumefantrine versus dihydroartemisinin-piperaquine for falciparum malaria: a longitudinal, randomized trial in young Ugandan children. Clin Infect Dis. 2009;49:1629-37.

5. Yeka A, Tibenderana J, Achan J, D'Alessandro U, Talisuna AO. Efficacy of quinine, artemether-lumefantrine and dihydroartemisinin-piperaquine as rescue treatment for uncomplicated malaria in Ugandan children. PLoS One. 2013;8:e53772.

6. Kamya MR, Yeka A, Bukirwa H, Lugemwa M, Rwakimari JB, Staedke SG, Talisuna AO, Greenhouse B, Nosten F, Rosenthal PJ, et al. Artemetherlumefantrine versus dihydroartemisinin-piperaquine for treatment of malaria: a randomized trial. PLoS Clin Trials. 2007;2:e20.

7. Byakika-Kibwika P, Achan J, Lamorde M, Karera-Gonahasa C, Kiragga AN, Mayanja-Kizza H, Kiwanuka N, Nsobya S, Talisuna AO, Merry C. Intravenous artesunate plus Artemisinin based combination therapy (ACT) or intravenous quinine plus ACT for treatment of severe malaria in Ugandan children: a randomized controlled clinical trial. BMC Infect Dis. 2017;17:794.

8. Kamya MR, Arinaitwe E, Wanzira H, Katureebe A, Barusya C, Kigozi SP, Kilama M, Tatem AJ, Rosenthal PJ, Drakeley C, et al. Malaria transmission, infection, and disease at three sites with varied transmission intensity in Uganda: implications for malaria control. Am J Trop Med Hyg. 2015;92:903-12.

9. Plowe CV, Djimde A, Bouare M, Doumbo O, Wellems TE. Pyrimethamine and proguanil resistance-conferring mutations in plasmodium falciparum dihydrofolate reductase: polymerase chain reaction methods for surveillance in Africa. Am J Trop Med Hyg. 1995;52:565-8. 
10. Zwetyenga J, Rogier C, Tall A, Fontenille D, Snounou G, Trape JF, Mercereau-Puijalon O. No influence of age on infection complexity and allelic distribution in plasmodium falciparum infections in Ndiop, a Senegalese village with seasonal, mesoendemic malaria. Am J Trop Med Hyg. 1998;59:726-35.

11. Borre MB, Dziegiel M, Hogh B, Petersen E, Rieneck K, Riley E, Meis JF, Aikawa M, Nakamura K, Harada M, et al. Primary structure and localization of a conserved immunogenic plasmodium falciparum glutamate rich protein (GLURP) expressed in both the preerythrocytic and erythrocytic stages of the vertebrate life cycle. Mol Biochem Parasitol. 1991;49:119-31.

12. Cattamanchi A, Kyabayinze D, Hubbard A, Rosenthal PJ, Dorsey G. Distinguishing recrudescence from reinfection in a longitudinal antimalarial drug efficacy study: comparison of results based on genotyping of msp-1, msp-2, and glurp. Am J Trop Med Hyg. 2003;68:133-9.

13. Lindegardh N, Annerberg A, White NJ, Day NP. Development and validation of a liquid chromatographic-tandem mass spectrometric method for determination of piperaquine in plasma stable isotope labeled internal standard does not always compensate for matrix effects. J Chromatogr B Anal Technol Biomed Life Sci. 2008;862:227-36.

14. Tarning J, Zongo I, Some FA, Rouamba N, Parikh S, Rosenthal PJ, Hanpithakpong W, Jongrak N, Day NP, White NJ, et al. Population pharmacokinetics and pharmacodynamics of piperaquine in children with uncomplicated falciparum malaria. Clin Pharmacol Ther. 2012;91:497-505.

15. Sambol NC, Yan L, Creek DJ, McCormack SA, Arinaitwe E, Bigira V, Wanzira H, Kakuru A, Tappero JW, Lindegardh N, et al. Population pharmacokinetics of Piperaquine in young Ugandan children treated with DihydroartemisininPiperaquine for uncomplicated malaria. Clin Pharmacol Ther. 2015;98:87-95.

16. Nkosi-Gondwe T, Robberstad B, Blomberg B, Phiri KS, Lange S. Introducing post-discharge malaria chemoprevention (PMC) for management of severe anemia in Malawian children: a qualitative study of community health workers' perceptions and motivation. BMC Health Serv Res. 2018;18:984.

17. Kwambai TK, Dhabangi A, Idro R, Opoka R, Kariuki S, Samuels AM, Desai M, van Hensbroek MB, John CC, Robberstad B, et al. Malaria chemoprevention with monthly dihydroartemisinin-piperaquine for the post-discharge management of severe anaemia in children aged less than 5 years in Uganda and Kenya: study protocol for a multi-Centre, two-arm, randomised, placebo-controlled, superiority trial. Trials. 2018;19:610.

18. Gondwe T, Robberstad B, Mukaka M, Lange S, Blomberg B, Phiri K. Delivery strategies for malaria chemoprevention with monthly dihydroartemisininpiperaquine for the post-discharge management of severe anaemia in children aged less than 5 years old in Malawi: a protocol for a cluster randomized trial. BMC Pediatr. 2018;18:238.

19. World Health Organisation. Guidelines for the Treatment of Malaria. 3rd ed; 2015.

\section{Publisher's Note}

Springer Nature remains neutral with regard to jurisdictional claims in published maps and institutional affiliations.

Ready to submit your research? Choose BMC and benefit from:

- fast, convenient online submission

- thorough peer review by experienced researchers in your field

- rapid publication on acceptance

- support for research data, including large and complex data types

- gold Open Access which fosters wider collaboration and increased citations

- maximum visibility for your research: over $100 \mathrm{M}$ website views per year

At $\mathrm{BMC}$, research is always in progress.

Learn more biomedcentral.com/submissions 